\title{
Hybrid Proofs of the $q$-Binomial Theorem and other identities
}

\author{
Dennis Eichhorn \\ Department of Mathematics \\ University of California \\ Irvine, Irvine, CA 92697-3875 \\ deichhor@math.uci.edu
}

\author{
James Mc Laughlin \\ Mathematics Department \\ West Chester University \\ West Chester, PA 19383 \\ jmclaughl@wcupa. edu
}

\author{
Andrew V. Sills \\ Department of Mathematical Sciences \\ 203 Georgia Avenue Room 3008 \\ Georgia Southern University \\ Statesboro, GA 30460-8093 \\ ASills@GeorgiaSouthern.edu
}

Submitted: Sep 10, 2010; Accepted: Feb 24, 2011; Published: Mar 11, 2011

Mathematics Subject Classifications: 11P84, 11P81

\begin{abstract}
We give "hybrid" proofs of the $q$-binomial theorem and other identities. The proofs are "hybrid" in the sense that we use partition arguments to prove a restricted version of the theorem, and then use analytic methods (in the form of the Identity Theorem) to prove the full version.

We prove three somewhat unusual summation formulae, and use these to give hybrid proofs of a number of identities due to Ramanujan.

Finally, we use these new summation formulae to give new partition interpretations of the Rogers-Ramanujan identities and the Rogers-Selberg identities.
\end{abstract}

\section{Introduction}

The proof of a $q$-series identity, whether a series-to-series identity such as the second iterate of Heine's transformation (see (4.1) below), a basic hypergeometric summation formula such as the $q$-Binomial Theorem (see (2.1)) or one of the Rogers-Ramanujan identities (see (S14) below), generally falls into one of two broad camps.

In the one camp, there are a variety of analytic methods. These include (but are certainly not limited to) elementary $q$-series manipulations (as in the proof of the BaileyDaum summation formula on page 18 of [15]), the use of difference operators (as in 
Gasper and Rahman's derivation of a bibasic summation formula [14]), the use of Bailey pairs and WP-Bailey pairs (see, for example, [7, 29, 31]), determinant methods (for example, $[17,26]$ ), constant term methods (such as in [4, Chap. 4]), polynomial finitization/generalization of infinite identities (as in [28]), an extension of Abel's Lemma (see [8, Chap. 7]), algorithmic methods such as the $q$-Zeilberger algorithm (as in $[12,19]$ ), matrix inversions (including those of Carlitz [11] and Krattenthaler [20]), $q$-Lagrange inversion (see $[2,16]$ ), Engel expansions (see $[5,6]$ ) and several other classical methods, including "Cauchy's Method" [18] and Abel's lemma on summation by parts [13].

In the other camp there are a variety of combinatorial or bijective proofs. Rather than attempt any classification of the various bijective proofs, we refer the reader to Pak's excellent survey [21] of bijective methods, with its extensive bibliography.

In the present paper we use a "hybrid" method to prove a number of basic hypergeometric identities. The proofs are "hybrid" in the sense that we use partition arguments to prove a restricted version of the theorem, and then use analytic methods (in the form of the Identity Theorem) to prove the full version.

We also prove three somewhat unusual summation formulae, and use these to give hybrid proofs of a number of identities due to Ramanujan. Finally, we use these new summation formulae to give new partition interpretations of the Rogers-Ramanujan identities and the Rogers-Selberg identities.

\section{A Hybrid Proof of the $q$-Binomial Theorem}

In this section we give a hybrid proof of the $q$-Binomial Theorem,

$$
\sum_{n=0}^{\infty} \frac{(a ; q)_{n} z^{n}}{(q ; q)_{n}}=\frac{(a z ; q)_{\infty}}{(z ; q)_{\infty}}
$$

Lemma 1. Let $k \geq 4$ and $r, s$ be fixed positive integers with $0<r<s<r+s<k$. For each positive integer $n$ and each integer $m \geq(r+k) n$, let $A_{n}(m)$ denote the number of partitions of $m$ with

- the part $r$ occurring exactly $n$ times,

- distinct parts from $\{s, s+k, s+2 k, \ldots, s+(n-1) k\}$,

- possibly repeating parts from $\{k, 2 k, 3 k, \ldots, n k\}$, with the part $n k$ occurring at least once.

Likewise, let $B_{n}(m)$ denote the number of partitions of $m$ into exactly $n$ parts, with

- distinct parts $\equiv r+s(\bmod k)$, with the part $r+s$ not appearing,

- possibly repeating parts $\equiv r(\bmod k)$, with the part $r$ not appearing.

Then

$$
A_{n}(m)=B_{n}(m)
$$

THE ELECTRONiC JOURNAL OF COMBINATORICS 18 (2011), \#P60 
Proof. We will exhibit injections between the two sets of partitions. We may represent a partition of $m$ of the type counted by $A_{n}(m)$ as

$$
m=\sum_{j=1}^{n} m_{j}(j k)+\sum_{j=0}^{n-1} \delta_{j}(j k+s)+n(r),
$$

where the parts are displayed in parentheses, and the multiplicities satisfy $m_{n} \geq 1, m_{j} \geq 0$ for $1 \leq j \leq n-1$, and $\delta_{j} \in\{0,1\}$. Upon applying the identity $\sum_{j=1}^{t} j y_{j}=\sum_{j=1}^{t} \sum_{i=j}^{t} y_{i}$ to the sums containing $j$, we get

$$
m=\left(m_{n} k+\delta_{n-1} s+r\right)+\sum_{j=1}^{n-1}\left(k \sum_{i=j}^{n} m_{i}+k \sum_{i=j}^{n-1} \delta_{i}+\delta_{j-1} s+r\right) .
$$

Here the parts of the new partition are displayed inside parentheses, and it is not difficult to recognize this partition as one of the type counted by $B_{n}(m)$.

On the other hand, we may represent a partition of $m$ of the type counted by $B_{n}(m)$ as

$$
m=\sum_{j=1}^{n}\left(p_{j} k+\delta_{j} s+r\right)
$$

with $1 \leq p_{1} \leq p_{2} \leq \cdots \leq p_{n}, \delta_{j} \in\{0,1\}$, and if $\delta_{i}=\delta_{i+1}=1$, then $p_{i}<p_{i+1}$. We also label the $p_{j}$ so that if $p_{i} k+\delta_{i} s+r>p_{j} k+\delta_{j} s+r$, then $i>j$ (in particular, this labeling means $p_{j+1}-p_{j}-\delta_{j} \geq 0$ for $\left.1 \leq j \leq n-1\right)$. We rewrite the above sum for $m$ as

$$
\begin{aligned}
m & =n[r]+\delta_{n}[s] \\
& +\left(p_{n}-p_{n-1}-\delta_{n-1}\right)[k]+\delta_{n-1}[k+s] \\
& +\left(p_{n-1}-p_{n-2}-\delta_{n-2}\right)[2 k]+\delta_{n-2}[2 k+s] \\
& +\left(p_{n-2}-p_{n-3}-\delta_{n-3}\right)[3 k]+\delta_{n-3}[3 k+s] \\
& \vdots \\
& +\left(p_{3}-p_{2}-\delta_{2}\right)[(n-2) k]+\delta_{2}[(n-2) k+s] \\
& +\left(p_{2}-p_{1}-\delta_{1}\right)[(n-1) k]+\delta_{1}[(n-1) k+s] \\
& +p_{1}[n k] .
\end{aligned}
$$

This is a partition of the type counted by $A_{n}(m)$, where this time the parts are displayed inside []'s.

It is not difficult to see that these transformations give injections between the two sets of partitions and the result is proved.

Graphically, we may describe these transformations as follows. In each case, we start with the usual Ferrers diagram of the partition.

It can be seen that the largest part in a partition counted by $A_{n}(m)$ has size $n k$, so such a partition can be regarded as consisting of $n$ columns, each of width $k$. The first 
step is to distribute the $n$ parts of size $r$ so that one $r$ is at the bottom of each of these $n$ columns. We then form a new partition whose parts are the columns of this intermediate partition (we might call it the $k$-block conjugate of this partition). This new partition is easily seen to be a partition of the type counted by $B_{n}(m)$.

If we start with a partition of the type counted by $B_{n}(m)$, the first step is to strip away a part of size $r$ from each of the $n$ parts. We then form the $k$-block conjugate of the remaining partition, add in the $n$ parts of size $r$, and what results is a partition of the type counted by $A_{n}(m)$.

We illustrate these transformations with two partitions of $26 k+4 s+5 r$ (with $n=5$ ). The partition with parts $5 k, 4 k+s, 4 k, 4 k, 3 k+s, 2 k, 2 k, k+s, k, s, r, r, r, r, r$ is one of those counted by $A_{5}(26 k+4 s+5 r)$. Its Ferrers diagram follows, and we show how it is transformed into the partition with parts $9 k+s+r, 7 k+s+r, 5 k+r, 4 k+r+s$ and $k+r+s$, which is a partition of the type counted by $B_{5}(26 k+4 s+5 r)$.

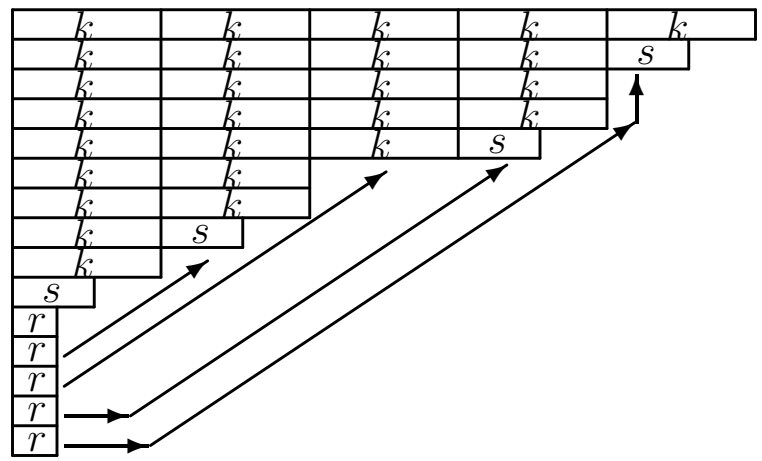

Figure 1. Place one part of size $r$ at the bottom of each of the 5 columns of width $k$.

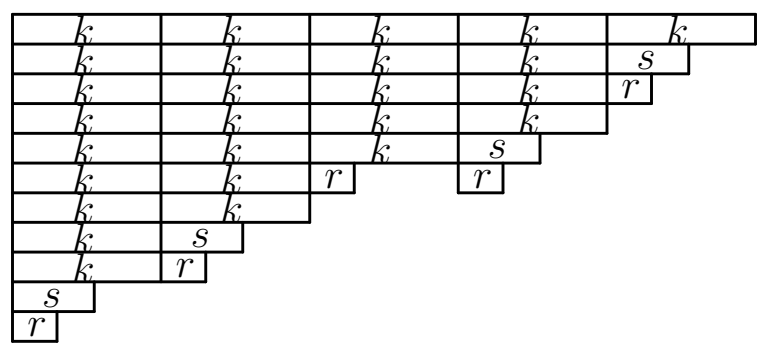

Figure 2. Now form the $k$-block conjugate of this partition.

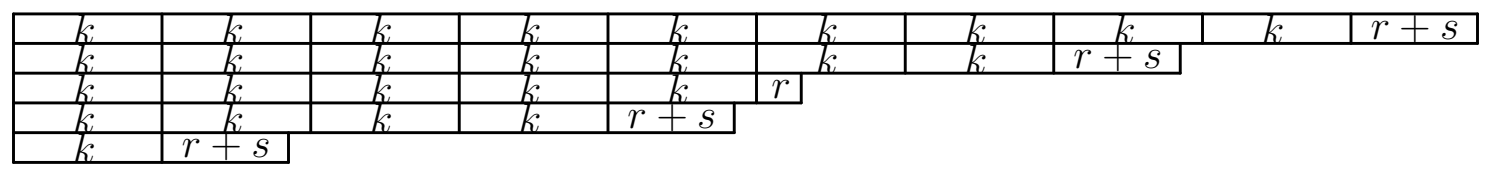

Figure 3 . This is a partition of the type counted by $B_{5}(26 k+4 s+5 r)$.

These steps are easily seen to be reversible. 
Lemma 2. Let $k \geq 4$ be a fixed integer and let $r$ and $s$ be fixed integers such that $0<r<s<r+s<k$. Then

$$
\sum_{n=0}^{\infty} \frac{\left(-q^{s} ; q^{k}\right)_{n}\left(q^{r+k}\right)^{n}}{\left(q^{k} ; q^{k}\right)_{n}}=\frac{\left(-q^{s+r+k} ; q^{k}\right)_{\infty}}{\left(q^{r+k} ; q^{k}\right)_{\infty}}
$$

Proof. The generating function for the sequence $A_{n}(m)$ is given by

$$
\frac{\left(-q^{s} ; q^{k}\right)_{n}\left(q^{r+k}\right)^{n}}{\left(q^{k} ; q^{k}\right)_{n}}=\sum_{m \geq(r+k) n} A_{n}(m) q^{m}
$$

Thus

$$
\begin{aligned}
1+\sum_{n=1}^{\infty} \frac{\left(-q^{s} ; q^{k}\right)_{n}\left(q^{r+k}\right)^{n}}{\left(q^{k} ; q^{k}\right)_{n}} & =1+\sum_{n=1}^{\infty} \sum_{m \geq(r+k) n} A_{n}(m) q^{m} \\
& =1+\sum_{n=1}^{\infty} \sum_{m \geq(r+k) n} B_{n}(m) q^{m} \\
& =1+\sum_{m \geq(r+k)} B(m) q^{m},
\end{aligned}
$$

where $B(m)$ counts the number of partitions of $m$ with

- distinct parts $\equiv r+s(\bmod k)$, with the part $r+s$ not appearing,

- possibly repeating parts $\equiv r(\bmod k)$, with the part $r$ not appearing.

It is clear that

$$
1+\sum_{m \geq(r+k)} B(m) q^{m}=\frac{\left(-q^{s+r+k} ; q^{k}\right)_{\infty}}{\left(q^{r+k} ; q^{k}\right)_{\infty}}
$$

and the result now follows.

We now give a proof of the $q$-Binomial Theorem.

Theorem 1. Let $a$, $z$ and $q$ be complex numbers with $|z|,|q|<1$. Then

$$
\sum_{n=0}^{\infty} \frac{(a ; q)_{n} z^{n}}{(q ; q)_{n}}=\frac{(a z ; q)_{\infty}}{(z ; q)_{\infty}}
$$

Proof. By (2.2), if $k$ and $m$ are positive integers with $k \geq 4$, and $r$ and $s$ are integers with $0<r<s m<s m+r<m k$, then

$$
\sum_{n=0}^{\infty} \frac{\left(-q^{s m} ; q^{k m}\right)_{n}\left(q^{r+k m}\right)^{n}}{\left(q^{k m} ; q^{k m}\right)_{n}}=\frac{\left(-q^{s m+r+k m} ; q^{k m}\right)_{\infty}}{\left(q^{r+k m} ; q^{k m}\right)_{\infty}}
$$


Fix an $m$-th root of $q$, denoted $q^{1 / m}$, and replace $q$ with $q^{1 / m}$ to get

$$
\sum_{n=0}^{\infty} \frac{\left(-q^{s} ; q^{k}\right)_{n}\left(q^{r / m+k}\right)^{n}}{\left(q^{k} ; q^{k}\right)_{n}}=\frac{\left(-q^{s+r / m+k} ; q^{k}\right)_{\infty}}{\left(q^{r / m+k} ; q^{k}\right)_{\infty}}
$$

Now let $m$ take the values $1,2,3, \ldots$, so that the identity

$$
\sum_{n=0}^{\infty} \frac{\left(-q^{s} ; q^{k}\right)_{n}\left(z q^{k}\right)^{n}}{\left(q^{k} ; q^{k}\right)_{n}}=\frac{\left(-q^{s+k} z ; q^{k}\right)_{\infty}}{\left(z q^{k} ; q^{k}\right)_{\infty}}
$$

holds for $z \in\left\{q^{r / m}: m \geq 1\right\}$. By continuity this identity also holds for $z=1$, the limit

of this sequence. Hence, by the Identity Theorem, (2.4) holds for $|z|<|q|^{-k}$. Replace $z$ with $z / q^{k}$ and we get that

$$
\sum_{n=0}^{\infty} \frac{\left(-q^{s} ; q^{k}\right)_{n} z^{n}}{\left(q^{k} ; q^{k}\right)_{n}}=\frac{\left(-q^{s} z ; q^{k}\right)_{\infty}}{\left(z ; q^{k}\right)_{\infty}}
$$

holds for $|z|<1$ and $1<s<k$.

Next, fix a $k$-th root of $q$, denoted $q^{1 / k}$, replace $q$ with $q^{1 / k}$ in $(2.5)$ to get that

$$
\sum_{n=0}^{\infty} \frac{\left(-q^{s / k} ; q\right)_{n} z^{n}}{(q ; q)_{n}}=\frac{\left(-q^{s / k} z ; q\right)_{\infty}}{(z ; q)_{\infty}}
$$

Set $s=2$ and let $k$ take the values $4,5,6, \ldots$ to get that

$$
\sum_{n=0}^{\infty} \frac{(a ; q)_{n} z^{n}}{(q ; q)_{n}}=\frac{(a z ; q)_{\infty}}{(z ; q)_{\infty}}
$$

holds for $a \in\left\{-q^{2 / k}: k \geq 4\right\}$ and $|z|<1$. By continuity, (2.7) also holds for $a=-1$, the limit point of this sequence. Thus, again by the Identity Theorem, (2.7) holds for all $a \in \mathbb{C}$ and all $z \in \mathbb{C}$ with $|z|<1$.

\section{Some Preliminary Summation Formulae}

Before coming to the proof of the next identities, we prove some preliminary lemmas.

Lemma 3. Let $|q|<1$ and $b \neq-q^{-n}$ for any positive integer $n$. Then if $m$ is any positive integer,

$$
\sum_{0 \leq a_{1} \leq a_{2} \leq \cdots \leq a_{n}} \frac{q^{m\left(a_{1}+a_{2}+\cdots+a_{n}\right)}}{\prod_{j=0}^{n-1} \prod_{k=1}^{m+1}\left(1+b q^{\left.j(m+1)+k+a_{j+1}\right)}\right.}=\frac{1}{\left(q^{m} ; q^{m}\right)_{n}(-b q ; q)_{m n}}
$$

where the sum is over all $n$-tuples $\left\{a_{1}, \ldots, a_{n}\right\}$ of integers that satisfy the stated inequality. 
Proof. We rewrite the left side of (3.1) as the nested sum

$$
\begin{gathered}
\sum_{a_{1} \geq 0} \frac{q^{m a_{1}}}{\prod_{k=1}^{m+1}\left(1+b q^{k+a_{1}}\right)} \sum_{a_{2} \geq a_{1}} \frac{q^{m a_{2}}}{\prod_{k=1}^{m+1}\left(1+b q^{(m+1)+k+a_{2}}\right)} \\
\ldots \sum_{a_{n-1} \geq a_{n-2}} \frac{q^{m a_{n-1}}}{\prod_{k=1}^{m+1}\left(1+b q^{\left.(n-2)(m+1)+k+a_{n-1}\right)}\right.} \\
\sum_{a_{n} \geq a_{n-1}} \frac{q^{m a_{n}}}{\prod_{k=1}^{m+1}\left(1+b q^{(n-1)(m+1)+k+a_{n}}\right)}
\end{gathered}
$$

Next, we note that if $p \geq 1$ is an integer, and none of the denominators following vanish, that

$$
\begin{aligned}
\sum_{a_{i} \geq a_{i-1}} \frac{q^{p m a_{i}}}{\prod_{k=1}^{m p+1}\left(1+c q^{k+a_{i}}\right)} & \\
=\frac{1}{1-q^{p m}} \sum_{a_{i} \geq a_{i-1}}\left[\frac{q^{p m a_{i}}}{\prod_{k=1}^{m p}\left(1+c q^{k+a_{i}}\right)}\right. & \left.-\frac{q^{p m\left(a_{i}+1\right)}}{\prod_{k=2}^{m p+1}\left(1+c q^{k+a_{i}}\right)}\right] \\
& =\frac{1}{1-q^{p m}} \frac{q^{p m a_{i-1}}}{\prod_{k=1}^{m p}\left(1+c q^{k+a_{i-1}}\right)}
\end{aligned}
$$

since the second sum telescopes. We now apply this result (with $p=1$ ) to the innermost sum at (3.2) to get that this sum has the value

$$
\frac{q^{m a_{n-1}}}{\left(1-q^{m}\right) \prod_{k=1}^{m}\left(1+b q^{\left.(n-1)(m+1)+k+a_{n-1}\right)}\right.},
$$

so that the next innermost sum at (3.2) becomes

$$
\sum_{a_{n-1} \geq a_{n-2}} \frac{q^{2 m a_{n-1}}}{\left(1-q^{m}\right) \prod_{k=1}^{2 m+1}\left(1+b q^{\left.(n-2)(m+1)+k+a_{n-1}\right)}\right.} .
$$

We apply (3.3) again, this time with $p=2$, to get that this sum has value

$$
\frac{q^{2 m a_{n-2}}}{\left(1-q^{m}\right)\left(1-q^{2 m}\right) \prod_{k=1}^{2 m}\left(1+b q^{\left.(n-2)(m+1)+k+a_{n-2}\right)}\right.} \text {. }
$$

This now results in the third innermost sum becomes

$$
\sum_{a_{n-2} \geq a_{n-3}} \frac{1}{\left(q^{m} ; q^{m}\right)_{2}} \frac{q^{3 m a_{n-2}}}{\prod_{k=1}^{3 m+1}\left(1+b q^{\left.(n-3)(m+1)+k+a_{n-2}\right)}\right.} .
$$

This process can be continued, so that after $n-1$ steps, the left side of (3.2) equals

$$
\begin{aligned}
& \sum_{a_{1} \geq 0} \frac{q^{m n a_{1}}}{\left(q^{m} ; q^{m}\right)_{n-1}} \prod_{k=1}^{n m+1}\left(1+b q^{k+a_{1}}\right) \\
& =\frac{q^{m n(0)}}{\left(q^{m} ; q^{m}\right)_{n-1}\left(1-q^{n m}\right) \prod_{k=1}^{n m}\left(1+b q^{k+0}\right)}=\frac{1}{\left(q^{m} ; q^{m}\right)_{n}(-b q ; q)_{n m}},
\end{aligned}
$$

giving the result. 
Lemma 4. Let $|q|<1$ and $b \neq-q^{-n}$ for any positive integer $n$. Then if $m$ is any positive integer,

$$
\sum_{0 \leq a_{1} \leq a_{2} \leq \cdots \leq a_{n}}^{\prime} \frac{q^{m\left(a_{1}+a_{2}+\cdots+a_{n}\right)}}{\prod_{j=0}^{n-1} \prod_{k=1}^{m+1}\left(1+b q^{\left.j m+k+a_{j+1}\right)}\right.}=\frac{1}{\left(q^{m} ; q^{m}\right)_{n}(-b q ; q)_{m n}}
$$

where the sum is over all n-tuples $\left\{a_{1}, \ldots, a_{n}\right\}$ of integers that satisfy the stated inequality, and the $\sum^{\prime}$ notation means that if $a_{i}=a_{i-1}$ for any $i$, then the factor $1+$ $b q^{(i-1) m+m+1+a_{i-1}}=1+b q^{i m+1+a_{i}}$ occurs just once in any product.

Proof. The proof is similar to the proof of Lemma 3. We rewrite the left side of (3.5) as the nested sum

$$
\begin{aligned}
\sum_{a_{1} \geq 0} \frac{q^{m a_{1}}}{\prod_{k=1}^{m+1}\left(1+b q^{k+a_{1}}\right)} & \sum_{a_{2} \geq a_{1}}^{\prime} \frac{q^{m a_{2}}}{\prod_{k=1}^{m+1}\left(1+b q^{m+k+a_{2}}\right)} \\
& \ldots \sum_{a_{n-1} \geq a_{n-2}}^{\prime} \frac{q^{m a_{n-1}}}{\prod_{k=1}^{m+1}\left(1+b q^{\left.(n-2) m+k+a_{n-1}\right)}\right.} \\
& \sum_{a_{n} \geq a_{n-1}}^{\prime} \frac{q^{m a_{n}}}{\prod_{k=1}^{m+1}\left(1+b q^{(n-1) m+k+a_{n}}\right)}
\end{aligned}
$$

Next, we note that if $p \geq 1$ is an integer, and the term $1+c q^{1+a_{i-1}}$ occurs in the next sum out, and none of the denominators following vanish, then

$$
\begin{aligned}
& \sum_{a_{i} \geq a_{i-1}}^{\prime} \frac{q^{p m a_{i}}}{\prod_{k=1}^{m p+1}\left(1+c q^{k+a_{i}}\right)} \\
& =\frac{q^{p m a_{i-1}}}{\prod_{k=2}^{m p+1}\left(1+c q^{k+a_{i-1}}\right)}+\sum_{a_{i} \geq a_{i-1}+1} \frac{q^{p m a_{i}}}{\prod_{k=1}^{m p+1}\left(1+c q^{k+a_{i}}\right)} \\
& =\frac{q^{p m a_{i-1}}}{\prod_{k=2}^{m p+1}\left(1+c q^{k+a_{i-1}}\right)}+\frac{1}{1-q^{p m}} \frac{q^{p m\left(a_{i}+1\right)}}{\prod_{k=1}^{m p}\left(1+c q^{k+a_{i-1}+1}\right)} \\
& =\frac{q^{p m a_{i-1}}}{\left(1-q^{p m}\right) \prod_{k=2}^{m p+1}\left(1+c q^{k+a_{i-1}}\right)},
\end{aligned}
$$

where the second equality follows from the same telescoping argument used in Lemma 3. We now apply this summation result repeatedly, starting with the innermost sum at (3.6) (with (with $p=1)$ ), to eventually arrive at the sum at (3.4) above, thus giving the result.

Lemma 5. Let $|q|<1$ and $b \neq-q^{-n}$ for any positive integer $n$. Then if $m$ is any positive integer,

$$
\sum_{0 \leq a_{1} \leq a_{2} \leq \cdots \leq a_{n}}^{\prime \prime} \frac{q^{m\left(a_{1}+a_{2}+\cdots+a_{n}\right)}}{\prod_{j=0}^{n-1} \prod_{k=0}^{m}\left(1+b q^{\left.j m+k+a_{j+1}\right)}\right.}=\frac{1}{\left(q^{m} ; q^{m}\right)_{n}(-b q ; q)_{m n}}
$$


where the sum is over all $n$-tuples $\left\{a_{1}, \ldots, a_{n}\right\}$ of integers that satisfy the stated inequality, and the $\sum^{\prime \prime}$ notation means that if $a_{i}=a_{i-1}$ for any $i$, then the factor $1+b q^{(i-1) m+m+a_{i-1}}=$ $1+b q^{i m+0+a_{i}}$ occurs just once in any denominator product, and in addition, if $a_{1}=0$, then the factor $1+b=1+b q^{0+0}$ does not appear in any denominator product.

Proof. The proof parallels the proof of Lemma 4, to get after $n-1$ steps, that the left side of (3.8) equals

$$
\begin{aligned}
& \sum_{a_{1} \geq 0}^{\prime \prime} \frac{q^{m n a_{1}}}{\left(q^{m} ; q^{m}\right)_{n-1} \prod_{k=0}^{n m}\left(1+b q^{k+a_{1}}\right)} \\
& =\frac{q^{m n(0)}}{\left(q^{m} ; q^{m}\right)_{n-1} \prod_{k=1}^{n m}\left(1+b q^{k+0}\right)}+\sum_{a_{1} \geq 1} \frac{q^{m n a_{1}}}{\left(q^{m} ; q^{m}\right)_{n-1} \prod_{k=0}^{n m}\left(1+b q^{k+a_{1}}\right)} \\
& =\frac{1}{\left(q^{m} ; q^{m}\right)_{n-1}(-b q ; q)_{m n}}+\frac{q^{m n(1)}}{\left(q^{m} ; q^{m}\right)_{n-1}\left(1-q^{m n}\right) \prod_{k=0}^{n m-1}\left(1+b q^{k+1}\right)} \\
& =\frac{1}{\left(q^{m} ; q^{m}\right)_{n}(-b q ; q)_{n m}}
\end{aligned}
$$

\section{Hybrid proofs of some $q$-series Identities}

We recall the second iterate of Heine's transformation (see [3, page 38]).

$$
\sum_{n=0}^{\infty} \frac{(a, b ; q)_{n}}{(c, q ; q)_{n}} t^{n}=\frac{(c / b, b t ; q)_{\infty}}{(c, t ; q)_{\infty}} \sum_{n=0}^{\infty} \frac{(a b t / c, b ; q)_{n}}{(b t, q ; q)_{n}}\left(\frac{c}{b}\right)^{n} .
$$

We will give a hybrid proof of a special case (set $c=0$, replace $a$ with $-a$ and $b$ with $-b q / t$, and finally let $t \rightarrow 0$ ) of this identity.

\section{Theorem 2.}

$$
\sum_{n=0}^{\infty} \frac{(-a ; q)_{n} b^{n} q^{n(n+1) / 2}}{(q ; q)_{n}}=(-b q ; q)_{\infty} \sum_{n=0}^{\infty} \frac{(a b)^{n} q^{n^{2}}}{(q,-b q ; q)_{n}}
$$

Remark: A version of (4.2) was stated by Ramanujan, see for example [8, Entry 1.6.1, page 24]. Proofs of (4.2) have been given by Ramamani [22] and Ramamani and Venkatachaliengar [23]. A generalization of (4.2) was proved by Bhargava and Adiga [10], while Srivastava [30] showed that (4.2) follows as a special case of Heine's transformation, as described above. Lastly, a combinatorial proof of (4.2) has been given in [9] by Berndt, Kim and Yee.

Proof of Theorem 2. We will prove for all integers $r, s$ and $k$ satisfying $0<r<s<$ $r+s<k$, that

$$
\sum_{n=0}^{\infty} \frac{\left(-q^{s} ; q\right)_{n} q^{r n} q^{k n(n+1) / 2}}{\left(q^{k} ; q^{k}\right)_{n}}=\left(-q^{r+k} ; q^{k}\right)_{\infty} \sum_{n=0}^{\infty} \frac{q^{(s+r) n} q^{k n^{2}}}{\left(q^{k},-q^{r+k} ; q^{k}\right)_{n}}
$$


and (4.2) will then follow from the Identity Theorem, by an argument similar to that used in the proof of the $q$-Binomial Theorem.

The $n$-th term in the series on the left side of (4.3) may be regarded as the generating function for partitions with

- the part $r$ occurring exactly $n$ times,

- distinct parts from $\{s, s+k, s+2 k, \ldots, s+(n-1) k\}$,

- possibly repeating parts from $\{k, 2 k, 3 k, \ldots, n k\}$, with each part occurring at least once.

We consider the Ferrers diagram for such a partition, which may be regarded as having $n$ columns, each of width $k$. We first distribute the $n$ parts of size $r$ so that one such part is placed at the bottom of each column. We then take the $k$-block conjugate of this partition we get a partition into $n$ parts with

- distinct parts $\equiv s+r(\bmod k)$, with the part $s+r$ not appearing and a gap of at least $2 k$ between consecutive parts,

- distinct parts $\equiv r(\bmod k)$, with the parts $r+j k$ and $r+(j+1) k$ not appearing if the part $r+s+j k$ appears (here $j \geq 1$ ).

Once again, this operation of taking the $k$-block conjugate gives a bijection between these two sets of partitions. If we now sum over all $n$, we get all partitions with

- distinct parts $\equiv s+r(\bmod k)$, with the part $s+r$ not appearing and a gap of at least $2 k$ between consecutive parts,

- distinct parts $\equiv r(\bmod k)$, with the parts $r+j k$ and $r+(j+1) k$ not appearing if the part $r+s+j k$ appears (here $j \geq 1$ ).

Next, instead of considering partitions of this latter type where there are a total of $n$ parts, we consider instead partitions of this type containing exactly $n$ parts $\equiv r+s(\bmod k)$. In other words we consider partitions with

- exactly $n$ distinct parts $\equiv s+r(\bmod k)$, with the part $s+r$ not appearing and a gap of at least $2 k$ between consecutive parts,

- distinct parts $\equiv r(\bmod k)$, with the parts $r+j k$ and $r+(j+1) k$ not appearing if the part $r+s+j k$ appears (here $j \geq 1$ ). 
It is not difficult to see that the generating function for such partitions is

$$
\begin{gathered}
\sum_{0 \leq a_{1} \leq \cdots \leq a_{n}} \frac{q^{\left(r+s+\left(1+a_{1}\right) k\right)+\left(r+s+\left(3+a_{2}\right) k\right)+\cdots+\left(r+s+\left(2 n-1+a_{n}\right) k\right)}\left(-q^{r+k} ; q^{k}\right)_{\infty}}{\prod_{j=1}^{n}\left(1+q^{r+\left(2 j-1+a_{j}\right) k}\right)\left(1+q^{r+\left(2 j+a_{j}\right) k}\right)} \\
=\left(-q^{r+k} ; q^{k}\right)_{\infty} q^{(r+s) n} q^{k n^{2}} \\
\quad \times \sum_{0 \leq a_{1} \leq a_{2} \leq \cdots \leq a_{n}} \frac{q^{\left(a_{1}+a_{2}+\cdots+a_{n}\right) k}}{\prod_{j=1}^{n}\left(1+q^{r+\left(2 j-1+a_{j}\right) k}\right)\left(1+q^{r+\left(2 j+a_{j}\right) k}\right)} \\
=\left(-q^{r+k} ; q^{k}\right)_{\infty} \frac{q^{(r+s) n} q^{k n^{2}}}{\left(q^{k} ; q^{k}\right)_{n}\left(-q^{r+k} ; q^{k}\right)_{n}}
\end{gathered}
$$

where the last equality follows from (3.1) (with $m=1, b=q^{r}$ and $q$ replaced with $q^{k}$ ). Now summing over all $n$ gives (4.3), and (4.2) follows.

We now prove a pair of identities stated by Ramanujan ([8, Entry 1.5.1, page 23], a replaced with $a q$ ). Analytic proofs were given by Watson [32] and Andrews [1], and a combinatorial proof has been given in [9] by Berndt, Kim and Yee.

Theorem 3. If $|q|<1$ and $a \neq-q^{-2 n}$ for any integer $n>0$, then

$$
\begin{aligned}
\sum_{n=0}^{\infty} \frac{a^{n} q^{n^{2}+n}}{(q ; q)_{n}} & =\left(-a q^{2} ; q^{2}\right)_{\infty} \sum_{n=0}^{\infty} \frac{a^{n} q^{n^{2}+2 n}}{\left(q^{2} ; q^{2}\right)_{n}\left(-a q^{2} ; q^{2}\right)_{n}} \\
& =\left(-a q^{3} ; q^{2}\right)_{\infty} \sum_{n=0}^{\infty} \frac{a^{n} q^{n^{2}+n}}{\left(q^{2} ; q^{2}\right)_{n}\left(-a q^{3} ; q^{2}\right)_{n}}
\end{aligned}
$$

Proof. We will prove only the first identity, as the proof of the second is very similar. We will first show, for all integers $0<r<k$, that

$$
\sum_{n=0}^{\infty} \frac{q^{r n} q^{k\left(n^{2}+n\right)}}{\left(q^{k} ; q^{k}\right)_{n}}=\left(-q^{r+2 k} ; q^{2 k}\right)_{\infty} \sum_{n=0}^{\infty} \frac{q^{r n} q^{k\left(n^{2}+2 n\right)}}{\left(q^{2 k} ; q^{2 k}\right)_{n}\left(-q^{r+2 k} ; q^{2 k}\right)_{n}}
$$

The $n$-th term in the series on the left side of (4.5) may be interpreted as the generating function for partitions with

- the part $r$ occurring exactly $n$ times,

- repeating parts from $\{k, 2 k, 3 k, \ldots, n k\}$, with each part occurring at least twice.

We once again consider the Ferrers diagram for such a partition, which also may be regarded as having $n$ columns, each of width $k$. We first distribute the $n$ parts of size $r$ so that one such part is placed at the bottom of each column. We then take the $k$-block conjugate of this partition we get a partition into $n$ parts with

- distinct parts $\equiv r(\bmod k)$, with the parts $r$ and $r+k$ not appearing and a gap of at least $2 k$ between consecutive parts. 
If we now sum over all $n$, we get all partitions with

- distinct parts $\equiv r(\bmod k)$, with the parts $r$ and $r+k$ not appearing and a gap of at least $2 k$ between consecutive parts.

We consider instead partitions of this type containing exactly $n$ distinct parts $\equiv r+$ $k(\bmod 2 k)$, with the part $r+k$ not appearing, and distinct parts $\equiv r(\bmod 2 k)$, with the part $r$ not appearing and a gap of at least $2 k$ between any consecutive parts. (If there are no parts $\equiv r+k(\bmod 2 k)$, then the partition consists entirely of distinct parts $\equiv r(\bmod 2 k)$, with the part $r$ not appearing, and these partitions have generating function $\left.\left(-q^{r+2 k} ; q^{2 k}\right)_{\infty}\right)$. In other words we consider partitions with

- exactly $n$ distinct parts $\equiv r+k(\bmod 2 k)$, with the part $r+k$ not appearing and a gap of at least $2 k$ between consecutive parts,

- distinct parts $\equiv r(\bmod 2 k)$, with the part $r$ not appearing, and with the parts $r+(2 j-2) k$ and $r+2 j k$ not appearing if the part $r+(2 j-1) k$ appears (here $j \geq 2)$.

The generating function for such partitions is

$$
\begin{gathered}
\sum_{0 \leq a_{1} \leq \cdots \leq a_{n}}^{\prime} \frac{q^{\left(r+\left(3+2 a_{1}\right) k\right)+\left(r+\left(5+2 a_{2}\right) k\right)+\cdots+\left(r+\left(2 n+1+2 a_{n}\right) k\right)}\left(-q^{r+2 k} ; q^{2 k}\right)_{\infty}}{\prod_{j=2}^{n+1}\left(1+q^{r+\left(2 j-2+2 a_{j}\right) k}\right)\left(1+q^{r+\left(2 j+2 a_{j}\right) k}\right)} \\
=\left(-q^{r+2 k} ; q^{2 k}\right)_{\infty} q^{r n} q^{k\left(n^{2}+2 n\right)} \\
\times \sum_{0 \leq a_{1} \leq a_{2} \leq \cdots \leq a_{n}} \frac{q^{\left(a_{1}+a_{2}+\cdots+a_{n}\right) 2 k}}{\prod_{j=1}^{n}\left(1+q^{r+\left(j+a_{j}\right) 2 k}\right)\left(1+q^{r+\left(j+1+a_{j}\right) 2 k}\right)} \\
=\left(-q^{r+2 k} ; q^{2 k}\right)_{\infty} \frac{q^{r n} q^{k\left(n^{2}+2 n\right)}}{\left(q^{2 k} ; q^{2 k}\right)_{n}\left(-q^{r+2 k} ; q^{2 k}\right)_{n}}
\end{gathered}
$$

where the last equality follows from (3.5) (with $b=q^{r}, m=1$ and $q$ replaced with $q^{2 k}$ ). Now summing over all $n$ gives (4.5), and the first identity at(4.4) follows once again by the Identity Theorem.

The proof of the second identity is similar, except that instead of considering partitions with exactly $n$ parts $\equiv r+k(\bmod 2 k)$ with the part $r+k$ not appearing, we consider partitions with exactly $n$ parts $\equiv r(\bmod 2 k)$ with the part $r$ not appearing. The second identity at (4.4) then follows, after some minor technicalities.

Next we give a hybrid proof of a special case of another identity of Ramanujan (see Entry 1.4.17 on page 22 of [8]).

Theorem 4. If $|q|<1$ and $a, b \neq-q^{-n}$ for any positive integer $n$, then

$$
(-b q ; q)_{\infty} \sum_{n=0}^{\infty} \frac{a^{n} q^{n(n+1) / 2}}{(q ; q)_{n}(-b q ; q)_{n}}=(-a q ; q)_{\infty} \sum_{n=0}^{\infty} \frac{b^{n} q^{n(n+1) / 2}}{(q ; q)_{n}(-a q ; q)_{n}}
$$


Remark: In the more general identity stated by Ramanujan, the terms $(-a q ; q)_{n}$ and $(-b q ; q)_{n}$ above are replaced, respectively, with $(-a q ; q)_{m n}$ and $(-b q ; q)_{m n}$, where $m$ is any positive integer. A combinatorial proof of Ramanujan's identity has been given in [9] by Berndt, Kim and Yee.

Proof. We will show for all integers $r, s, k$ satisfying $0<r<s<k$ that

$$
\left(-q^{r+k} ; q^{k}\right)_{\infty} \sum_{n=0}^{\infty} \frac{q^{s n} q^{k n(n+1) / 2}}{\left(q^{k},-q^{r+k} ; q^{k}\right)_{n}}=\left(-q^{s+k} ; q^{k}\right)_{\infty} \sum_{n=0}^{\infty} \frac{q^{r n} q^{k n(n+1) / 2}}{\left(q^{k},-q^{s+k} ; q^{k}\right)_{n}},
$$

and the full result at (4.6) will follow once again from the Identity Theorem.

By (3.5) (with $m=1$ and $q$ replaced with $q^{k}$ ), the left side of (4.7) equals

$$
\begin{aligned}
\sum_{n=0}^{\infty} \sum_{0 \leq a_{1} \leq a_{2} \leq \cdots \leq a_{n}}^{\prime} \frac{q^{\sum_{j=1}^{n} s+\left(j+a_{j}\right) k}\left(-q^{r+k} ; q^{k}\right)_{\infty}}{\prod_{j=0}^{n-1}\left(1+q^{r+\left(j+1+a_{j+1}\right) k}\right)\left(1+q^{r+\left(j+2+a_{j+1}\right) k}\right)} \\
=\sum_{n=0}^{\infty} \sum_{0 \leq a_{1} \leq a_{2} \leq \cdots \leq a_{n}}^{\prime} \frac{q^{\sum_{j=1}^{n} s+\left(j+a_{j}\right) k}\left(-q^{r+k} ; q^{k}\right)_{\infty}}{\prod_{j=1}^{n}\left(1+q^{r+\left(j+a_{j}\right) k}\right)\left(1+q^{r+\left(j+1+a_{j}\right) k}\right)} .
\end{aligned}
$$

The $n$-th term of this latter series may be regarded as the generating function for partitions with

- exactly $n$ distinct parts $\equiv s(\bmod k)$, with the part $s$ not appearing,

- distinct parts $\equiv r(\bmod k)$, with the part $r$ not appearing, and with the parts $r+p k$ and $r+(p+1) k$ not appearing if the part $s+p k$ appears (here $p \geq 1$ ),

and so the entire series may be regarded as the generating function for partitions with

- distinct parts $\equiv s(\bmod k)$, with the part $s$ not appearing,

- distinct parts $\equiv r(\bmod k)$, with the part $r$ not appearing, and with the parts $r+p k$ and $r+(p+1) k$ not appearing if the part $s+p k$ appears (here $p \geq 1)$.

These conditions are equivalent to the conditions

- distinct parts $\equiv r(\bmod k)$, with the part $r$ not appearing,

- distinct parts $\equiv s(\bmod k)$, with the part $s$ not appearing, and with the parts $s+$ $(p-1) k$ and $s+p k$ not appearing if the part $r+p k$ appears (here $p \geq 1$ ). 
The generating function for such partitions containing exactly $n$ distinct parts $\equiv r(\bmod k)$ is

$$
\begin{aligned}
\sum_{0 \leq a_{1} \leq a_{2} \leq \cdots \leq a_{n}}^{\prime \prime} & \frac{q^{\sum_{j=1}^{n} r+\left(j+a_{j}\right) k}\left(-q^{s+k} ; q^{k}\right)_{\infty}}{\prod_{j=1}^{n}\left(1+q^{s+\left(j-1+a_{j}\right) k}\right)\left(1+q^{s+\left(j+a_{j}\right) k}\right)} \\
= & \sum_{0 \leq a_{1} \leq a_{2} \leq \cdots \leq a_{n}}^{\prime \prime} \frac{q^{\sum_{j=1}^{n} r+\left(j+a_{j}\right) k}\left(-q^{s+k} ; q^{k}\right)_{\infty}}{\prod_{j=0}^{n-1}\left(1+q^{s+\left(j+a_{j+1}\right) k}\right)\left(1+q^{s+\left(j+1+a_{j+1}\right) k}\right)} \\
& =\frac{\left(-q^{s+k} ; q^{k}\right)_{\infty} q^{r n} q^{k n(n+1) / 2}}{\left(q^{k},-q^{s+k} ; q^{k}\right)_{n}}
\end{aligned}
$$

where the last equality follows from (3.8) (with $m=1, q$ replaced with $q^{k}$, and $b=q^{s}$ ). The identity at (4.7) now follows upon summing over all $n$.

\section{Some New Partitions Identities Deriving from Identities of Rogers-Ramanujan-Slater type}

Lemmas 3 - 5 allow us to derive new partition interpretations of some well-known analytic identities.

\subsection{The Rogers-Ramanujan Identities}

The following identities appear in Slater's paper [29] (S14 refers to the identity numbered (14) in Slater's paper [29], and similarly for other identities labelled below):

$$
\begin{aligned}
\sum_{n=0}^{\infty} \frac{q^{n^{2}+n}}{(q ; q)_{n}} & =\frac{1}{\left(q^{2}, q^{3} ; q^{5}\right)_{\infty}}, \\
\left(-q^{2} ; q^{2}\right)_{\infty} \sum_{n=0}^{\infty} \frac{q^{n^{2}+2 n}}{\left(q^{4} ; q^{4}\right)_{n}} & =\frac{1}{\left(q^{2}, q^{3} ; q^{5}\right)_{\infty}}, \\
\left(-q ; q^{2}\right)_{\infty} \sum_{n=0}^{\infty} \frac{q^{n^{2}+n}}{\left(q^{2} ; q^{2}\right)_{n}\left(-q ; q^{2}\right)_{n+1}} & =\frac{1}{\left(q^{2}, q^{3} ; q^{5}\right)_{\infty}} .
\end{aligned}
$$

Each of these identities had also previously been proven by Rogers [24]. The equality of the three left sides of these equations easily follow from Theorem 3, and in fact they could also be proved directly from the summation formulae in Lemmas 4 and 5 .

Perhaps more interesting is the result of interpreting the left sides of S16 and S94 using the summation formula in Lemma 3. As is well known, the identity at S14 (The Second Rogers-Ramanujan Identity) implies that if $A(n)$ denotes the number of partitions of $n$ into distinct parts with no 1's and a gap of at least 2 between consecutive parts, and $B(n)$ denotes the number of partitions of $n$ into parts $\equiv 2,3(\bmod 5)$, then $A(n)=B(n)$ for all positive integers $n$. Lemma 3 now lets us describe two other sets of partitions of 
each positive integer $n$ which are also equinumerous with the sets of partitions counted by $A(n)$ and $B(n)$.

Theorem 5. For a positive integer $n$, let $A(n)$ denotes the number of partitions of $n$ into distinct parts with no 1's and a gap of at least 2 between consecutive parts, and let $B(n)$ denote the number of partitions of $n$ into parts $\equiv 2,3(\bmod 5)$.

Let $C(n)$ denote the number of partitions of $n$ into distinct parts with no 1 's appearing, such that if $o_{j}$ is the $j$-th odd part (where we order the parts in ascending order), then the even parts $o_{j}+2 j-3$ and $o_{j}+2 j-1$ do not appear.

Let $D(n)$ denote the number of partitions of $n$ into distinct parts with no 1 's appearing, such that if $e_{j}$ is the $j$-th even part (where again we order the parts in ascending order), then the odd parts $e_{j}+2 j-1$ and $e_{j}+2 j+1$ do not appear.

Then

$$
A(n)=B(n)=C(n)=D(n) .
$$

Proof. From what has been said already about the equality of the three left sides at S14, S16 and S94, all that is necessary is to show that

$$
\begin{aligned}
\left(-q^{2} ; q^{2}\right)_{\infty} \sum_{n=0}^{\infty} \frac{q^{n^{2}+2 n}}{\left(q^{4} ; q^{4}\right)_{n}} & =\sum_{n=0}^{\infty} C(n) q^{n} \\
\left(-q ; q^{2}\right)_{\infty} \sum_{n=0}^{\infty} \frac{q^{n^{2}+n}}{\left(q^{2} ; q^{2}\right)_{n}\left(-q ; q^{2}\right)_{n+1}} & =\sum_{n=0}^{\infty} D(n) q^{n} .
\end{aligned}
$$

We do this for the second identity only, since the proof for the former follows similarly. By Lemma 3, with $q$ replaced with $q^{2}, m=1$ and $b=q$,

$$
\begin{aligned}
\left(-q ; q^{2}\right)_{\infty} & \sum_{n=0}^{\infty} \frac{q^{n^{2}+n}}{\left(q^{2} ; q^{2}\right)_{n}\left(-q ; q^{2}\right)_{n+1}}=\left(-q^{3} ; q^{2}\right)_{\infty} \sum_{n=0}^{\infty} \frac{q^{n^{2}+n}}{\left(q^{2} ; q^{2}\right)_{n}\left(-q^{3} ; q^{2}\right)_{n}} \\
& =\sum_{n=0}^{\infty} \sum_{0 \leq a_{1} \leq a_{2} \leq \cdots \leq a_{n}} \frac{q^{\left(2+2 a_{1}\right)+\left(4+2 a_{2}\right)+\cdots+\left(2 n+2 a_{n}\right)}\left(-q^{3} ; q^{2}\right)_{\infty}}{\prod_{j=1}^{n}\left(1+q^{4 j-1+2 a_{j}}\right)\left(1+q^{4 j+1+2 a_{j}}\right)} .
\end{aligned}
$$

This last series is the generating function for partitions into distinct parts, with no 1's appearing, and such that if $2 j+2 a_{j}$ is the $j$-th even part, then the odd parts $\left(2 j+2 a_{j}\right)+$ $2 j-1$ and $\left(2 j+2 a_{j}\right)+2 j+1$ do not appear. This is precisely the partitions of an integer $n$ counted by $D(n)$.

As an example we consider the nine partitions of 15 counted by $B(15), C(15)$ and $D(15)$. Those counted by $B(15)$ are

$$
\{3,2,2,2,2,2,2\},\{3,3,3,2,2,2\},\{3,3,3,3,3\},\{7,2,2,2,2\},\{7,3,3,2\},
$$

$$
\{8,3,2,2\},\{8,7\},\{12,3\},\{13,2\},
$$

those counted by $C(15)$ are

$$
\{7,5,3\},\{8,5,2\},\{9,4,2\},\{9,6\},\{10,5\},\{11,4\},\{12,3\},\{13,2\},\{15\},
$$


while those counted by $D(15)$ are

$$
\{7,5,3\},\{7,6,2\},\{8,4,3\},\{8,7\},\{10,5\},\{11,4\},\{12,3\},\{13,2\},\{15\} .
$$

Note that $D(15)$ does not count, for example, $\{8,5,2\}$ (since $e_{1}=2$ and $e_{1}+2(1)+1=$ 5 ), while $C(15)$ does not count, for example, $\{7,6,2\}$ (since $o_{1}=7$ and $o_{1}+2(1)-3=6$ ).

Three partner identities which also appear in Slater's paper [29] and which were also previously proven by Rogers [24] are the following:

$$
\begin{aligned}
\sum_{n=0}^{\infty} \frac{q^{n^{2}}}{(q ; q)_{n}} & =\frac{1}{\left(q, q^{4} ; q^{5}\right)_{\infty}}, \\
\left(-q^{2} ; q^{2}\right)_{\infty} \sum_{n=0}^{\infty} \frac{q^{n^{2}}}{\left(q^{2},-q^{2} ; q^{2}\right)_{n}} & =\frac{1}{\left(q, q^{4} ; q^{5}\right)_{\infty}} \\
\left(-q ; q^{2}\right)_{\infty} \sum_{n=0}^{\infty} \frac{q^{n^{2}+n}}{\left(q^{2} ; q^{2}\right)_{n}\left(-q ; q^{2}\right)_{n}} & =\frac{1}{\left(q, q^{4} ; q^{5}\right)_{\infty}} .
\end{aligned}
$$

The equality of the three left sides of these equations once again easily follow from the summation formulae in Lemmas 4 and 5. The identity S18 (The First Rogers-Ramanujan Identity) also has a well-known interpretation in terms of partitions, namely, that if $A(n)$ denotes the number of partitions of $n$ into distinct parts with a gap of at least 2 between consecutive parts, and $B(n)$ denotes the number of partitions of $n$ into parts $\equiv 1,4(\bmod 5)$, then $A(n)=B(n)$ for all positive integers $n$.

As with the previous three identities, Lemma 3 implies two new partition identities.

Theorem 6. For a positive integer $n$, let $A(n)$ denotes the number of partitions of $n$ into distinct parts a gap of at least 2 between consecutive parts, and let $B(n)$ denote the number of partitions of $n$ into parts $\equiv 1,4(\bmod 5)$.

Let $C(n)$ denote the number of partitions of $n$ into distinct parts, such that if $o_{j}$ is the $j$-th odd part (where we order the parts in ascending order), then the even parts $o_{j}+2 j-3$ and $o_{j}+2 j-1$ do not appear.

Let $D(n)$ denote the number of partitions of $n$ into distinct parts, such that if $e_{j}$ is the $j$-th even part (where again we order the parts in ascending order), then the odd parts $e_{j}+2 j-3$ and $e_{j}+2 j-1$ do not appear.

Then

$$
A(n)=B(n)=C(n)=D(n)
$$

Proof. Once again, all that is necessary is to show that

$$
\begin{aligned}
\left(-q^{2} ; q^{2}\right)_{\infty} \sum_{n=0}^{\infty} \frac{q^{n^{2}}}{\left(q^{4} ; q^{4}\right)_{n}} & =\sum_{n=0}^{\infty} C(n) q^{n} \\
\left(-q ; q^{2}\right)_{\infty} \sum_{n=0}^{\infty} \frac{q^{n^{2}+n}}{\left(q^{2} ; q^{2}\right)_{n}\left(-q ; q^{2}\right)_{n}} & =\sum_{n=0}^{\infty} D(n) q^{n}
\end{aligned}
$$


As in the proof of the previous theorem, we do this for the second identity only, since the proof for the former follows similarly. By Lemma 3, with $q$ replaced with $q^{2}, m=1$ and $b=1 / q$,

$$
\begin{aligned}
\left(-q ; q^{2}\right)_{\infty} & \sum_{n=0}^{\infty} \frac{q^{n^{2}+n}}{\left(q^{2} ; q^{2}\right)_{n}\left(-q ; q^{2}\right)_{n}} \\
& =\sum_{n=0}^{\infty} \sum_{0 \leq a_{1} \leq a_{2} \leq \cdots \leq a_{n}} \frac{q^{\left(2+2 a_{1}\right)+\left(4+2 a_{2}\right)+\cdots+\left(2 n+2 a_{n}\right)}\left(-q ; q^{2}\right)_{\infty}}{\prod_{j=1}^{n}\left(1+q^{4 j-3+2 a_{j}}\right)\left(1+q^{4 j-1+2 a_{j}}\right)} .
\end{aligned}
$$

This last series is the generating function for partitions into distinct parts, such that if $2 j+2 a_{j}$ is the $j$-th even part, then the odd parts $\left(2 j+2 a_{j}\right)+2 j-3$ and $\left(2 j+2 a_{j}\right)+2 j-1$ do not appear. This is precisely the partitions of an integer $n$ counted by $D(n)$.

This time, as an example, we consider the six partitions of 10 counted by $B(10), C(10)$ and $D(10)$. Those counted by $B(10)$ are

$$
\{1,1,1,1,1,1,1,1,1,1\},\{4,1,1,1,1,1,1\},\{4,4,1,1\},\{6,1,1,1,1\},\{6,4\},\{9,1\},
$$

those counted by $C(10)$ are

$$
\{5,4,1\},\{6,4\},\{7,3\},\{8,2\},\{9,1\},\{10\},
$$

while those counted by $D(10)$ are

$$
\{6,3,1\},\{6,4\},\{7,3\},\{8,2\},\{9,1\},\{10\} .
$$

Note that $D(10)$ does not count $\{5,4,1\}$ (since $e_{1}=4$ and $e_{1}+2(1)-1=5$ ), while $C(10)$ does not count $\{6,3,1\}$ (since $o_{2}=3$ and $o_{2}+2(2)-1=6$ ).

\subsection{The Rogers-Selberg Identities}

Before coming to the Rogers - Selberg identities, we recall that the union of the partitions $\pi$ and $\lambda$, denoted $\pi \cup \lambda$, is the partition whose parts are those of $\pi$ and $\lambda$ together, arranged in non-increasing order. For example,

$$
\{4,3,3,2,2,1\} \cup\{5,4,3,2,2,1,1\}=\{5,4,4,3,3,3,2,2,2,2,1,1,1\} .
$$

A bipartition of a positive integer $n$ is an ordered pair of partitions $(\pi, \lambda)$ such that $\pi \cup \lambda$ is a partition of $n$. Note that $\pi$ or $\lambda$ may be empty.

The following identity was proved by Rogers [24] and also later by Selberg [27] and Slater [29]:

$$
(-q ; q)_{\infty} \sum_{n=0}^{\infty} \frac{q^{2 n^{2}}}{\left(q^{2} ; q^{2}\right)_{n}(-q ; q)_{2 n}}=\frac{1}{\left(q, q^{2}, q^{5}, q^{6} ; q^{7}\right)_{\infty}} .
$$

We may interpret this identity as follows. 
Theorem 7. For a positive integer $n$, let $A(n)$ denote the number of partitions of $n$ into parts $\equiv \pm 1, \pm 2(\bmod 7)$.

Let $B(n)$ denote the number of bipartitions $(\pi, \lambda)$ of $n$, where $\pi$ is a partition into distinct even parts with a gap of at least 4 between consecutive parts, and $\lambda$ is a partition into distinct parts such that if $e_{j}$ is the $j$-th part in $\pi$ (where we order the parts in ascending order), then the parts $e_{j} / 2, e_{j} / 2+1$ and $e_{j} / 2+2$ are not present in $\lambda$.

Let $C(n)$ denote the number of bipartitions $(\pi, \mu)$ of $n$, where $\pi$ is as above, and $\mu$ is a partition into distinct parts such that if $e_{j}$ is the $j$-th part in $\pi$ (where, as above, we order the parts in ascending order), then the parts $e_{j} / 2+j-1, e_{j} / 2+j$ and $e_{j} / 2+j+1$ are not present in $\mu$.

Then

$$
A(n)=B(n)=C(n) .
$$

Proof. The right side of $(\mathbf{S 3 3})$ clearly gives $\sum_{n=0}^{\infty} A(n) q^{n}$. By Lemmas 3 and 4 , respectively, with $b=1$ and $m=2$ in each case,

$$
\begin{aligned}
&(-q ; q)_{\infty} \frac{q^{2 n^{2}}}{\left(q^{2} ; q^{2}\right)_{n}(-q ; q)_{2 n}} \\
&=\sum_{0 \leq a_{1} \leq a_{2} \leq \cdots \leq a_{n}} \frac{q^{\left(2+2 a_{1}\right)+\left(6+2 a_{2}\right)+\cdots+\left(4 n-2+2 a_{n}\right)}(-q ; q)_{\infty}}{\prod_{j=1}^{n}\left(1+q^{3 j-2+a_{j}}\right)\left(1+q^{3 j-1+a_{j}}\right)\left(1+q^{3 j+a_{j}}\right)} \\
&=\sum_{0 \leq a_{1} \leq a_{2} \leq \cdots \leq a_{n}}^{\prime} \frac{q^{\left(2+2 a_{1}\right)+\left(6+2 a_{2}\right)+\cdots+\left(4 n-2+2 a_{n}\right)}(-q ; q)_{\infty}}{\prod_{j=1}^{n}\left(1+q^{2 j-1+a_{j}}\right)\left(1+q^{2 j+a_{j}}\right)\left(1+q^{2 j+1+a_{j}}\right)}
\end{aligned}
$$

Upon noting that the $j$-th addend in the exponent of $q$ in each of the two multiple sums above is $e_{j}=4 j-2+2 a_{j}$, it can be seen that summing the first of these sums over all $n$ gives $\sum_{n=0}^{\infty} C(n) q^{n}$, while summing the second over all $n$ gives $\sum_{n=0}^{\infty} B(n) q^{n}$.

Remark: It is not until $n=14$ do we reach an integer for which there is a difference in the bipartitions counted by $B(n)$ and those counted by $C(n):(\{8,4\},\{4\})$ is counted by $C(14)$ but not $B(14)$ (since $\left.e_{2} / 2=4\right)$, and $(\{6,2\},\{6\})$ is counted by $B(14)$ but not $C(14)$ (since $e_{2} / 2+2+1=3+2+1=6$ ).

A similar analysis (with $b=q$ and $m=2$ in Lemmas 3 and 4 ) of the next identity, also due independently to Rogers [25], Selberg [27] and Slater [29],

$$
\left(-q^{2} ; q\right)_{\infty} \sum_{n=0}^{\infty} \frac{q^{2 n^{2}+2 n}}{\left(q^{2} ; q^{2}\right)_{n}\left(-q^{2} ; q\right)_{2 n}}=\frac{1}{\left(q^{2}, q^{3}, q^{4}, q^{5} ; q^{7}\right)_{\infty}}
$$

leads to the following partition interpretation.

Theorem 8. For a positive integer $n$, let $A(n)$ denote the number of partitions of $n$ into parts $\equiv \pm 2, \pm 3(\bmod 7)$.

Let $B(n)$ denote the number of bipartitions $(\pi, \lambda)$ of $n$, where $\pi$ is a partition into distinct even parts greater than 2 with a gap of at least 4 between consecutive parts, and $\lambda$ 
is a partition into distinct parts greater than 1 such that if $e_{j}$ is the $j$-th part in $\pi$ (where we order the parts in ascending order), then the parts $e_{j} / 2, e_{j} / 2+1$ and $e_{j} / 2+2$ are not present in $\lambda$.

Let $C(n)$ denote the number of bipartitions $(\pi, \mu)$ of $n$, where $\pi$ is as above, and $\mu$ is a partition into distinct parts greater than 1 such that if $e_{j}$ is the $j$-th part in $\pi$ (where, as above, we order the parts in ascending order), then the parts $e_{j} / 2+j-1, e_{j} / 2+j$ and $e_{j} / 2+j+1$ are not present in $\mu$.

Then

$$
A(n)=B(n)=C(n) .
$$

Remark: In this case it is not until $n=19$ do we reach an integer for which there is a difference in the bipartitions counted by $B(n)$ and those counted by $C(n):(\{10,4\},\{5\})$ is counted by $C(19)$ but not $B(19)$ (since $e_{2} / 2=5$ ), and $(\{8,4\},\{7\})$ is counted by $B(19)$ but not $C(19)$ ( since $\left.e_{2} / 2+2+1=4+2+1=7\right)$.

Lastly, an analysis (with $b=1$ and $m=2$ in Lemmas 3 and 4 ) of the remaining Rogers-Selberg-Slater identity ([25], [27] and [29]),

$$
(-q ; q)_{\infty} \sum_{n=0}^{\infty} \frac{q^{2 n^{2}+2 n}}{\left(q^{2} ; q^{2}\right)_{n}(-q ; q)_{2 n}}=\frac{1}{\left(q, q^{3}, q^{4}, q^{6} ; q^{7}\right)_{\infty}}
$$

leads to the following result.

Theorem 9. For a positive integer $n$, let $A(n)$ denote the number of partitions of $n$ into parts $\equiv \pm 1, \pm 3(\bmod 7)$.

Let $B(n)$ denote the number of bipartitions $(\pi, \lambda)$ of $n$, where $\pi$ is a partition into distinct even parts greater than 2 with a gap of at least 4 between consecutive parts, and $\lambda$ is a partition into distinct parts such that if $e_{j}$ is the $j$-th part in $\pi$ (where we order the parts in ascending order), then the parts $e_{j} / 2-1, e_{j} / 2$ and $e_{j} / 2+1$ are not present in $\lambda$.

Let $C(n)$ denote the number of bipartitions $(\pi, \mu)$ of $n$, where $\pi$ is as above, and $\mu$ is a partition into distinct parts such that if $e_{j}$ is the $j$-th part in $\pi$ (where, as above, we order the parts in ascending order), then the parts $e_{j} / 2+j-2, e_{j} / 2+j-1$ and $e_{j} / 2+j$ are not present in $\mu$.

Then

$$
A(n)=B(n)=C(n) .
$$

This time, it is not until $n=18$ do we reach an integer for which there is a difference in the bipartitions counted by $B(n)$ and those counted by $C(n):(\{10,4\},\{4\})$ is counted by $C(18)$ but not $B(18)$ (since $e_{2} / 2-1=5-1=4$ ), and $(\{8,4\},\{6\}$ ) is counted by $B(18)$ but not $C(18)$ (since $e_{2} / 2+2=4+2=6$ ).

\section{Concluding Remarks}

In the bijective part of the hybrid proofs given in the paper, we have used only the simplest of all bijections, namely, conjugation. It is likely that other bijections will lead to hybrid proofs of other basic hypergeometric identities. 
The fact that Ramanujan's identity Entry 1.4.17 generalizes the identity in Theorem 4 (see the remark following Theorem 4) suggests that it may be possible to generalize the summation formulae in Section 3.

\section{References}

[1] G. E. Andrews, q-identites of Auluck, Carlitz and Rogers, Duke Math. J. 33 (1966), 575-581.

[2] G. E. Andrews, Identities in combinatorics. II. A q-analog of the Lagrange inversion theorem, Proc. Amer. Math. Soc. 53 (1975), no. 1, 240-245.

[3] G. E. Andrews, The Theory of Partitions, Addison-Wesley, 1976, Reissued Cambridge, 1998.

[4] G .E. Andrews, q-series: their development and application in analysis, number theory, combinatorics, physics, and computer algebra, CBMS Regional Conference Series in Mathematics, 66, Amer. Math. Soc., Providence, RI, 1986.

[5] G. E. Andrews, A. Knopfmacher and J. Knopfmacher, Engel expansions and the Rogers-Ramanujan identities, J. Number Theory 80 (2000), no. 2, 273-290.

[6] G. E. Andrews, A. Knopfmacher and P. Paule, An infinite family of Engel expansions of Rogers-Ramanujan type, Adv. in Appl. Math. 25 (2000), no. 1, 2-11.

[7] G. E. Andrews and A. Berkovich, The WP-Bailey tree and its implications, J. London Math. Soc. (2) 66 (2002), no. 3, 529-549.

[8] G. E. Andrews and B. C. Berndt, Ramanujan's Lost Notebook, Part II, Springer, 2009.

[9] B. C. Berndt, B. Kim and A. J. Yee, Ramanujan's lost notebook: Combinatorial proofs of identities associated with Heine's transformation or partial theta functions, J. Combin. Theory Ser. A 117 (2010), no. 7, 957-973.

[10] S. Bhargava and C. Adiga, A basic hypergeometric transformation of Ramanujan and a generalization, Indian J. Pure Appl. Math. 17 (1986), no. 3, 338-342.

[11] L. Carlitz, Some inverse relations, Duke Math J. 40 (1973), 893-901.

[12] W. Y. C. Chen, Q.-H. Hou and Y.-P. Mu, Non-terminating basic hypergeometric series and the q-Zeilberger algorithm, Proc. Edinb. Math. Soc. (2) 51 (2008), no. 3, 609-633.

[13] W. Chu, Abel's lemma on summation by parts and basic hypergeometric series, Adv. in Appl. Math. 39 (2007), no. 4, 490-514.

[14] G. Gasper and M. Rahman, An indefinite bibasic summation formula and some quadratic, cubic and quartic summation and transformation formulae, Canad. J. Math. 42 (1990), 1-27.

[15] G. Gasper and M. Rahman, Basic hypergeometric series, With a foreword by Richard Askey. Second edition. Encyclopedia of Mathematics and its Applications, 96, Cambridge University Press, Cambridge, 2004. xxvi+428 pp. 
[16] I. Gessel and D. Stanton, Applications of q-Lagrange inversion to basic hypergeometric series, Trans. Amer. Math. Soc. 277 (1983), no. 1, 173-201.

[17] N. S. S. Gu and H. Prodinger, One-Parameter Generalizations of Rogers-Ramanujan Type Identities, Adv. in Appl. Math. 45 (2010), no. 2, 149-196.

[18] F. Jouhet and M. Schlosser, Another proof of Bailey's ${ }_{6} \psi_{6}$ summation, Aequationes Math. 70 (2005), no. 1-2, 43-50.

[19] T. H. Koornwinder, On Zeilberger's algorithm and its q-analogue, J. Comput. Appl. Math. 48 (1993), no. 1-2, 91-111.

[20] C. Krattenthaler, A new matrix inverse, Proc. Amer. Math. Soc. 124 (1996), no. 1, 47-59.

[21] I. Pak, Partition bijections, a survey, Ramanujan J. 12 (2006), no. 1, 5-75.

[22] V. Ramamani, Some identities conjectured by Srinivasa Ramanujan found in his lithographed notes connected with partition theory and elliptic modular functions their proofs - interconnection with various other topics in the theory of numbers and some generalizations thereon, PhD thesis (1970), University of Mysore, Mysore.

[23] V. Ramamani and K. Venkatachaliengar, On a partition theorem of Sylvester, Michigan Math. J. 19 (1972), 137-140.

[24] L. J. Rogers, Second memoir on the expansion of certain infinite products, Proc. London Math. Soc. 25 (1894), 318-343.

[25] L. J. Rogers, On two theorems of combinatory analysis and some allied identities, Proc. London Math. Soc. 16 (1917), 315-336.

[26] I. Schur, Ein Beitrag zur additiven Zahlentheorie und zur Theorie der Kettenbrüchen, in Gesammelte Abhandlungen. Band II, Springer-Verlag, Berlin-New York (1973), 117-136. Originally in Sitzungsberichte der Preussischen Akadamie der Wissenschaften, Physikalisch-Mathematische Klasse (1917), 302-321.

[27] A. Selberg, Über einige arithmetische Identitäten, Avrandlinger Norske Akad. 8, 1936.

[28] A. V. Sills, Finite Rogers-Ramanujan type identities, Electronic J. Combin. 10 \#R13 (2003), 122 pp.

[29] L. J. Slater, Further identities of the Rogers-Ramanujan type, Proc. London Math. Soc. 54 (1952), 147-167.

[30] H. M. Srivastava, A note on a generalization of a q-series transformation of Ramanujan, Proc. Japan Acad. Ser. A Math. Sci. 63 (1987), no. 5, 143-145.

[31] S. O. Warnaar, Extensions of the well-poised and elliptic well-poised Bailey lemma, Indag. Math. (N.S.) 14 (2003), no. 3-4, 571-588.

[32] G. N. Watson, A note on Lerch's functions, Quart. J. Math., Oxford Ser. 8 (1937), 43-47. 